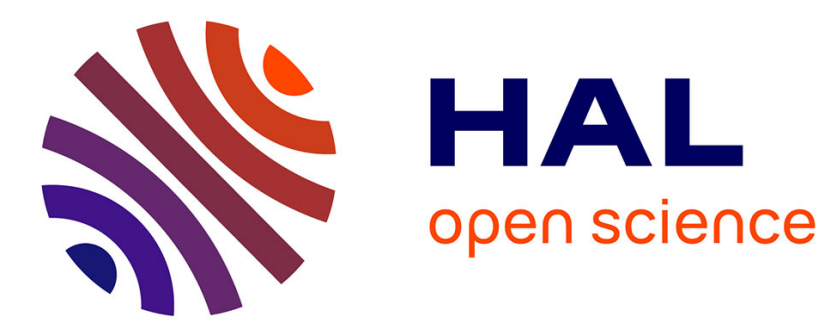

\title{
An Efficient Implementation of Tiled Polymorphic Temporal Media
}

\author{
Simon Archipoff
}

\section{To cite this version:}

Simon Archipoff. An Efficient Implementation of Tiled Polymorphic Temporal Media. ICFP FARM, ACM SIGPAN, Sep 2015, Vancouver, Canada. 10.1145/2808083.2808089 . hal-01214101

\section{HAL Id: hal-01214101 \\ https://hal.science/hal-01214101}

Submitted on 12 Oct 2015

HAL is a multi-disciplinary open access archive for the deposit and dissemination of scientific research documents, whether they are published or not. The documents may come from teaching and research institutions in France or abroad, or from public or private research centers.
L'archive ouverte pluridisciplinaire HAL, est destinée au dépôt et à la diffusion de documents scientifiques de niveau recherche, publiés ou non, émanant des établissements d'enseignement et de recherche français ou étrangers, des laboratoires publics ou privés. 


\title{
An Efficient Implementation of Tiled Polymorphic Temporal Media
}

\author{
Simon Archipoff * \\ simon.archipoff@u-bordeaux.fr \\ LaBRI \\ Université de Bordeaux
}

\begin{abstract}
Tiled Polymorphic Temporal Media (TPTM) are a convenient way to describe, compose and encode multimedia streams. This paper presents a TPTM encoding that allows simple and efficient implementation of both composition and rendering. In particular, an on-the-fly rendering procedure is provided in order to handle infinite (lazy) TPTM.
\end{abstract}

\section{Introduction}

In the context of computerized music, but also in the context of systems producing temporal media, the purpose of computation not only concerns what values are to be computed as in classical programming, but also when these values are to be produced or rendered.

In these fields, temporal media (sequences of media values that evolve with time, such as audio or video) play a prominent role. In [2], a polymorphic temporal media algebra is defined, allowing to combine them either in sequence or in parallel. Presumably, these operators are sufficient. However, the analysis of common functions, such as temporal media synchronization, suggests that temporal media may be abstracted further.

In order to compose of two audio sequences $m_{1}$ and $m_{2}$, we have to take into account the content of $m_{1}$ and $m_{2}$ in order to make a consistent audio

\footnotetext{
*Partially funded by INEDIT, ANR-12-CORD-oog.
}

alignment. A silence $d$ may have to be added to the composition in order to synchronize $m_{1}$ and $m_{2}$. More precisely, such a composition would be $(d:+$ : $\left.m_{1}\right):=: m_{2}$ or $m_{1}:=:\left(d:+: m_{2}\right)$, where $:+$ denotes the sequential composition and $:=$ : denotes the parallel composition. Of course, an abstraction of the form

$$
\lambda m_{1} m_{2} \rightarrow\left(d:+: m_{1}\right):=: m_{2}
$$

would be meaningless since the value $d$ depends on both $m_{1}$ and $m_{2}$. Instead, the correct abstraction of the composition of two audio sequences $m_{1}$ and $m_{2}$ can be defined by:

$$
\begin{aligned}
\lambda m_{1} m_{2} \rightarrow & \text { let } d=\text { alignOffset } m_{1} m_{2} \\
\text { in if } d>0 & \text { then }\left(d:+: m_{1}\right):=: m_{2} \\
& \text { else } m_{1}:=:\left((-d):+: m_{2}\right)
\end{aligned}
$$

where the function alignOffset performs a possibly complex analysis of the underlying musical structure induced by $m_{1}$ and $m_{2}$.

The synchronization data of two temporal media $m_{1}$ and $m_{2}$ can be independent one with the other, for instance for musical bars. This suggests that the underlying abstract structure that may relevantly describes these audio sequences should not only consists in the ordered audio frames themselves, that form the basis of the audio sequences, but also in a number of synchronization marks al- 
lowing to adequately align these audio sequences one with each other.

As a matter of fact, most audio toolboxes that are available nowadays propose mechanisms to enrich audio or musical sequences with such a kind of synchronization markers. Yet, while these markers are defined (in a quite ad hoc way) at the interface level, their definition, manipulation and transformation at the programmatic level is far from being clearly understood.

Despite an apparent simplicity, defining and handling adequate synchronization marks over temporal media is not easy: how many synchronization marks should we allow? This immediately raises the question of representing the (possibly many and heterogeneous) time scales the temporal media are to be defined on. Such a time scale can be for instance: seconds, bars, beats, events... Even more, as soon as interaction appears, this poses the problem of building systems that are globally asynchronous (event driven) and locally synchronous (almost continuous).

The tiled modeling and tiled programming approach that is recalled and implemented in this paper is an attempt to formalize, not only at the programmatic level but also at the modeling level, the interplay between temporal media values: what they are, and their synchronization marks: how they should be used. In particular, it aims at providing sufficiently rich abstract description of temporal media types so that as we shall see, one can easily define a meaningful abstract composition process:

$$
\lambda m_{1} m_{2} \rightarrow m_{1} \% m_{2}
$$

where $\%$ is the tiled composition operator. The resulting domain specific language is both versatile and efficient.

In a first part of this paper we will recall some of the work done in this field. Then we will present our main contribution: a new implementation of Tiled Polymorphic Temporal Media. Last, we will study how this implementation compare to other ones considering algorithmic complexity, performances, and finally expressiveness. As an illustra- tion of expressiveness, we present a set of primitives to convert Polymorphic temporal media to Tiled Polymorphic Temporal Media using our implementation.

\section{From PTM to TPTM}

In this section we give a brief survey of the existing approaches, from Hudak's Polymorphic Temporal Media (PTM) to the more recently proposed Tiled Polymorphic Temporal Media (TPTM).

An important concept with PTM and therefore TPTM is the notion of observational equivalence between two temporal media. The observational equivalence of $m_{1}$ and $m_{2}$, denoted by $m_{1} \equiv m_{2}$, means that $m_{1}$ and $m_{2}$ can not be distinguished during the rendering, no matter how they are combined with other temporal media.

\subsection{Polymorphic Temporal Media (PTM)}

A Hudak's Polymorphic Temporal Media [2] can be either a temporal media value lasting for some duration $d$, a sequential composition :+: or a parallel composition :=: of any two simpler media. Such operators are depicted in Figure 1 where, as in most figures, the horizontal axis represents the flow of time from left to right.

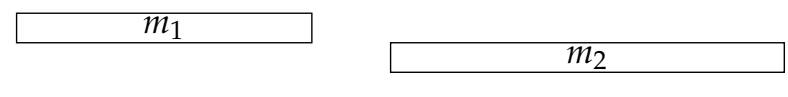

$$
m_{1} \text { and } m_{2}
$$

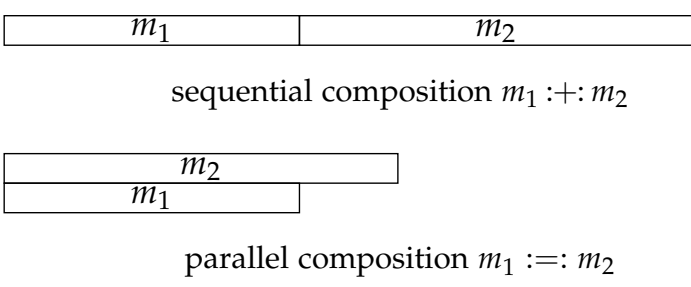

Figure 1: PTM syntax and semantics

Despite its simplicity, this model is used in computer music library such as Haskore [6] and Eu- 
terpea [3] (both written in Haskell). It has nice algebraic properties [2].

This model handles infinite temporal media thanks to laziness. For instance in $m=m_{1}:+: m, m$ is a valid and playable temporal media when $m_{1}$ is finite. About the implementation, the "on demand" semantics of Haskell spares the programmer a lot of work and makes the code very simple.

With the simplicity of this approach come two slight drawbacks. The first one concerns the semantics of the parallel composition, we have many ways to synchronize two temporal media in parallel (at their beginning, at their end, truncating the end of the longest...). We have to make an arbitrary choice: in Euterpea, the parallel composition synchronizes the two operands at their beginning, and the duration of the resulting media is the one of the longest operand. The second drawback is the lack of modularity mentioned in the introduction.

\subsection{Tiled Polymorphic Temporal Media (TPTM)}

Tiled Polymorphic Temporal Media proposed by Hudak and Janin [4] offers a simpler approach with a single tiled composition operator (denoted \%). With TPTM, the information about the synchronization is embedded in the media and is used in the tiled composition. It follows that both sequential and parallel compositions appear as particular cases of tiled composition.

Tiled PTM allows the decorrelation of the beginning and the end of the temporal media from their synchronization points. More precisely, a tile is a stream enriched with two particular points called pre $(\Upsilon)$ and post $(\downarrow)$, as depicted in Figure 2.

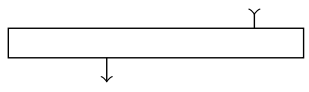

Figure 2: A tile

Then, given two tiles $t_{1}$ and $t_{2}$ encapsulating streams $s_{1}$ and $s_{2}$ respectively, we compute the composition $t_{1} \% t_{2}$ by synchronizing the post marker of $t_{1}$ with the pre marker of $t_{2}$, and mixing their underlying streams. The synchronization points of the resulting tile are the pre marker of $t_{1}$ and the post marker of $t_{2}$, as depicted in Figure 3. Thus, tiles $t_{1}$ and $t_{2}$ describe how $s_{1}$ and $s_{2}$ are aligned.
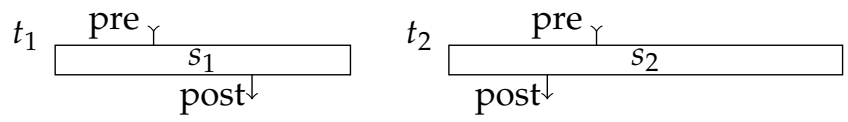

(a) Two stream descriptors enriched with pre and post synchronization points

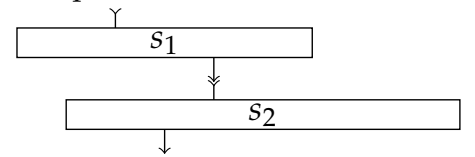

(b) When composing $t_{1}$ with $t_{2}$, the post synchronization point of $t_{1}$ is aligned with the pre synchronization point of $t_{2}$

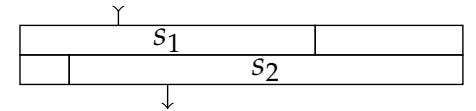

(c) The streams $s_{1}$ and $s_{2}$ are completed with silences and composed together to make a new tile

Figure 3: Tiles are enriched streams

\subsection{Derived Operators}

The model is rich enough so that we can easily define derived functions to compose tiles. The reset of a tile re $t$ is a tile of duration 0 with the post marker at the same place as the pre marker in $t$. It can be used to make parallel composition of tiles by synchronizing them at their pre marker. The coreset function co places the pre marker of a tile at the position of the post marker, making a tile of duration 0 . It is useful to synchronize two parallel tiles on their post marker. And the inv function swaps the pre and the post marker. These functions are depicted in Figure 4.

The inv function have a very interesting algebraic property. Under a few hypothesis on the underlying media, the set of tiles equipped with product $\%$ and the empty tile unit (of duration 0 ) forms an inverse monoid with regard to observational equiva- 


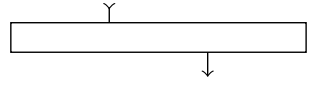

$t$

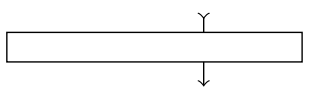

$\cot$

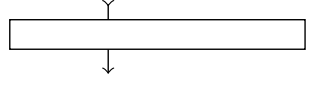

re $t$

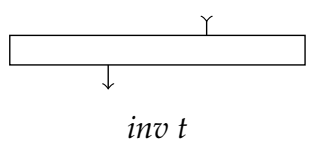

Figure 4: A tile $t$, and its reset, co-reset, and inverse

lence [4]. More precisely the product \% is associative, unit is neutral and for every tile $x$ there is a unique tile inv $x$ verifying:

$$
x \% \operatorname{inv} x \% x \equiv x
$$

$\operatorname{inv} x \% x \%$ inv $x \equiv \operatorname{inv} x$

\subsection{Parallel Operators}

Using those operators, we can define fork and join binary operators, two parallel compositions synchronizing tiles at their pre and post marker, respectively. The fork operator behaves somehow like the $:=$ : compositor of PTM. The semantics of these functions are depicted in Figure 5.

$$
\text { fork } t_{1} t_{2}=\text { re } t_{1} \% t_{2}
$$$$
\text { join } t_{1} t_{2}=t_{1} \% \text { co } t_{2}
$$

\subsection{Modeling Examples}

Tiled Polymorphic Temporal Media are convenient to handle media according to their underlying structures beginning or ending, which can be distinct from their literal beginning or ending. Possible applications of this property are detailed below.

\subsubsection{Adding a Pickup}

In music, an anacrusis, or pickup, is a brief introduction to a measure. Using TPTM, it is simple to add a pickup to a tile. Given a tile $t$ with a positive duration and nothing before the pre marker, it

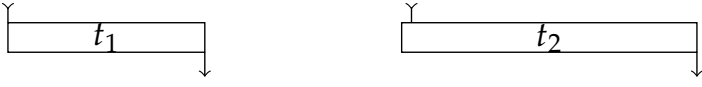

(a) $t_{1}$ and $t_{2}$

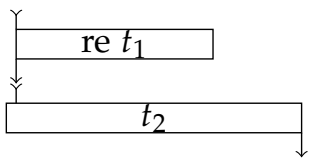

ret $t_{1} \% t_{2}$

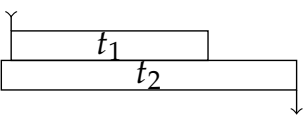

(b) fork $t_{1} t_{2}$

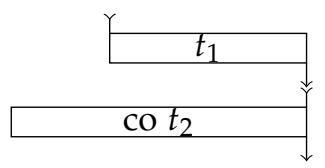

$t_{1} \% \cot _{2}$

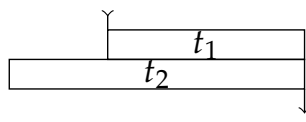

(c) join $t_{1} t_{2}$
Figure 5: The fork and join functions

is possible to add a pickup $p$ by computing co $p \% t$ as depicted in Figure 6.

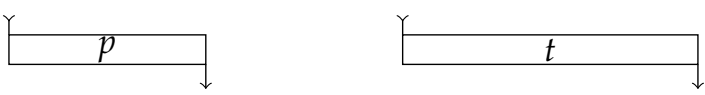

A tile $t$ and a pickup $p$

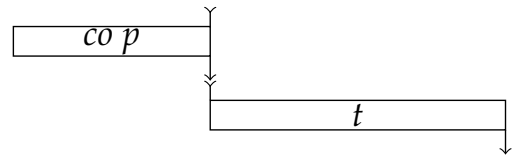

The co-reset of $p$ is composed with $t$

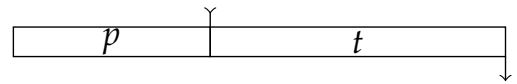

The tile $\operatorname{cop} \% t$ with its underlying media merged

Figure 6: Adding a pickup with tiles

In this example, we can see a case where TPTM are more modular than PTM. In $a \% t \% b$, if one wants to add something in $\mathrm{t}$, as long as the markers of $t$ are placed in a consistent way, neither $a$ nor $b$ will be impacted by this modification. 


\subsubsection{Uniform Handling of Language and Rhyth- mical Units}

As a more concrete application instance, tiles are convenient to modelize the relation between the lyrics and the music. Those two objects do not have the same logical structure. One is decomposed in verses, grammatical units, words,... the other in phrases, bars,... Nevertheless, they are both entangled in a song and TPTM can formalize these relations.

Here, we consider the Bob Dylan's song "Blowing in the wind". We can encode the lyrics by a list of tiles where the text embedded by each tile is determined by grammar, and the markers of those tiles by musical criteria. In Figure 7 the lyrics are split using grammatical criteria, the pre and post markers are placed according to the musical bars location.

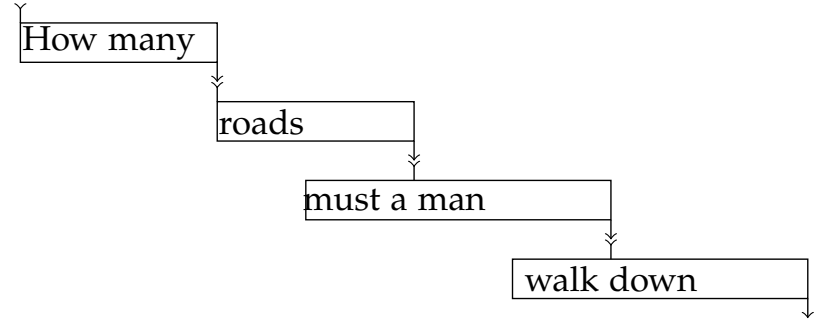

Figure 7: A song tiled by measures

We build a list of tiles with a duration of one measure. By packing these tiles four by four, we tile the verses of the song, as shown in Figure 8.

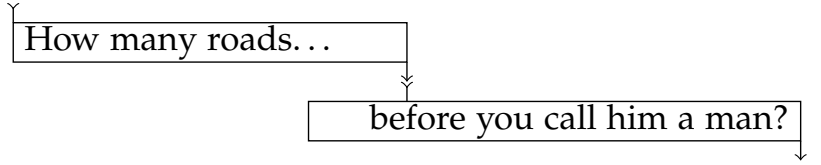

Figure 8: A song Tiled by verses of 4 measures

This illustrates the potential of TPTM to represent music at various time scales, and according to the different structures involved (natural language, measure, melody,...).

\section{Existing Implementations}

Implementing TPTM is not a trivial task. We have to satisfy the constraint of polymorphism, this implies to make minimal hypothesis on the tiled media. We also want to handle infinite data structure, and we want our tiles to be playable in real time.

\subsection{Tiles in Euterpea}

Hudak and Janin made a first implementation on top of Euterpea [4] by encapsulating Euterpea's Music type into a type Tile:

$$
\begin{aligned}
& \text { data Tile } a=\text { Tile }\{\text { preT }:: \text { Duration; } \\
& \text { post } T:: \text { Duration; } \\
& \text { musT :: Music a \} }
\end{aligned}
$$

with Duration implementing the set of rational numbers.

Simple and elegant, this implementation already allows to study the notion of tiled programming. However, it suffers from a lack of polymorphism, as primitives on tiles are implemented with primitives of Music type.

\subsection{The Syntactic Approach}

Other standalone implementations proposed by Bazin, Hudak and Janin $[1,5]$ separate clearly the out-of-time tile construction induced by tiled modeling and the in-time on-the-fly rendering. To this end, they encode tiles in a syntactic way.

This settles a set of primitives that allows both composition and rendering of tiles.

\subsection{Creation Primitives}

First, the out-of-time primitives used to produce tiles:

$$
\begin{aligned}
& \text { delay }:: \text { Duration } \rightarrow \text { Tile } a \\
& \text { event }:: a \rightarrow \text { Tile } a \\
& (\%):: \text { Tile } a \rightarrow \text { Tile } a \rightarrow \text { Tile } a \\
& \text { dur }:: \text { Tile } a \rightarrow \text { Duration }
\end{aligned}
$$


This syntax describes the two atomic tiles. The expression delay $d$ is an empty tile of duration $d$ which can be positive as well as negative, and event $e$ is a tile of null duration with a single event $e$. The Figure 9 shows two atomic tiles. In most figures the horizontal axis represent the time and the scale is respected. The dur function returns the duration of the tile, defined as the time distance between the pre and post markers. We observe that dur $(a \% b) \equiv d u r a+d u r b$. The \% operator allows the composition of those two base tiles.

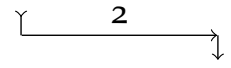

(a) The tile delay 2

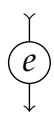

(b) The tile event $e$
Figure 9: Two simple tile

As delays can be negative, the temporality of the media does not have to be reflected in the description of the tile. For instance, in event $a \%$ delay $(-1) \%$ event $b$, the event $b$ will occur before the event $a$. Then, we can describe every tile by its sequence of events separated by back and forth delays, hence the resulting tiles are "zigzag" through time. An example of such a tile is depicted in Figure 10.

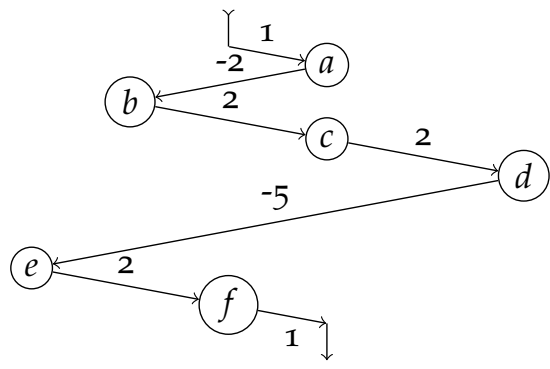

Figure 10: A tile "zigzag" $t$

With those construction primitives, we can implement the reset, co-reset, and inverse functions described earlier. These functions are depicted in Figure 4 .

$$
\begin{aligned}
\text { inv } t= & \text { let } d=\text { dur } t \\
& \text { in delay }(-d) \% t \% \text { delay }(-d) \\
r e t= & t \% \text { delay }(-(\text { dur } t)) \\
\cot t= & \text { delay }(-(\text { dur } t)) \% t
\end{aligned}
$$

\subsection{Rendering Primitives}

Obviously, zigzag tiles can not be rendered as they are. The first event to occur can be deep in the syntactic structure. For each tile, there is a normal form where there is no consecutive delays, and no negative delay between two events. Such a normal form is depicted in Figure 11.

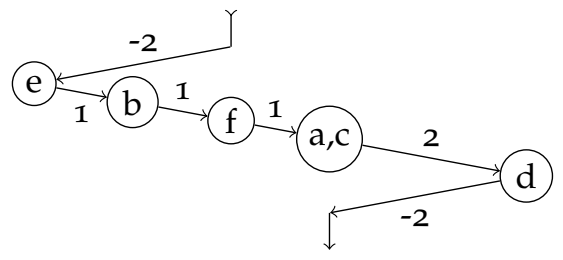

Figure 11: The tile $t$ normalized

One way to compute such a normal form is to define some primitives to extract the first events and the time distance to them:

$$
\begin{aligned}
& \text { first } E:: \text { Tile } a \rightarrow \text { MultiSet } a \\
& \text { firstD :: Tile } a \rightarrow \text { Maybe Duration }
\end{aligned}
$$

The first $E$ function returns the list (encoding a multiset) of all the first events (all the other are strictly later). And the firstD function returns the delay between the pre marker and the first events. Such a delay will not be defined if the tile have no event. That is the reason why first $D$ returns a value of type Maybe Duration. It can be either Just d, returned when the tile have events, or Nothing when there is no such event.

In order to play a tile, we need to compute the first events to occur, when they will occur, but also what comes afterward. We define the primitives headT and tailT to compute these. The headT function returns the tile composed of the first delay and the first simultaneous events, and tailT returns the remainder of the tile, as depicted in Figure 12. 
headT :: Tile $a \rightarrow$ Tile $a$

tailT $::$ Tile $a \rightarrow$ Tile $a$

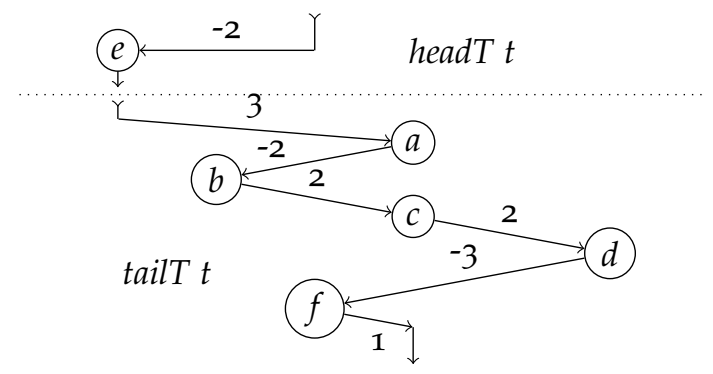

Figure 12: Same tile as in Figure 10 with the first event extracted

For any tile, we have the property:

$$
t \equiv \text { headT } t \% \text { tailT } t
$$

Those functions are named after the head and tail functions defined over lists because of the semantic similarity between them. Over any non empty list $l$ the following property is verified.

$$
l==\text { head } l: \text { tail } l
$$

The headT function can be defined in term of firstD and firstE:

$$
\begin{aligned}
& \text { headT } t= \\
& \text { case first } D t \text { of } \\
& \text { Nothing } \rightarrow \text { delay } 0 \\
& \text { Just } d \rightarrow \text { delay } d \% \text { fold ( } \lambda \text { te } \rightarrow t \% \text { event } e \text { ) } \\
& \text { (delay } 0) \\
& \text { (firstE } t \text { ) }
\end{aligned}
$$

As event only takes a single event, the multiset returned by first $E$ is reduced in a tile using tiled product and the standard Haskell function fold. This function takes as parameter an accumulation function (here of type Tile $e \rightarrow e \rightarrow$ Tile $e$ ) and a starting accumulator value (here delay 0 , a neutral).

\subsection{Observational Equivalence}

The simplest observational equivalence is the following: two tiles are considered equivalent when they have the same duration and describe the same marked sequence of timed events. Such a definition does not takes into account the properties of the events type, relevant from the observation. It is recursively implemented by:

$$
\begin{aligned}
& (\equiv):: \text { Eq e } \Rightarrow \text { Tile } e \rightarrow \text { Tile } e \rightarrow \text { Bool } \\
& (\equiv) t_{1} t_{2}= \\
& \text { case }\left(\text { first } E t_{1}, \text { first } t_{2}\right) \text { of } \\
& ([],[]) \rightarrow \text { dur } t_{1}==\text { dur } t_{2} \\
& (\text { le1,le } 2) \rightarrow \text { first } t_{1}==\text { first } t_{2} \\
& \\
& \wedge \text { first } t_{1}==\text { firstD } t_{2} \\
& \\
& \wedge \text { tailT } t_{1} \equiv \text { tailT } t_{2}
\end{aligned}
$$

The base case is when both tiles have no event, then it checks if they are equal in duration. The recursive case checks if the two tiles have the same first events, at the same time, and if their remainders (their tails) are equivalent.

\section{Heaped Tiles}

Here, we present a new approach using balanced data structure and making the duration of the tile independent of the delays used to build it. For this, we define a tile as a duration (of type Duration) and a set of timed events. Such a set is implemented by a mergeable heap, here Sleator and Tarjan's skew heap [7].

The implementation of the tile is given by:

data Tile $e=$ Tile Duration (SHeap e)

with Duration the duration of the tile and SHeap e a set of timed events.

The skew heap of events is a binary tree where node are labeled by a delay and embed some events.

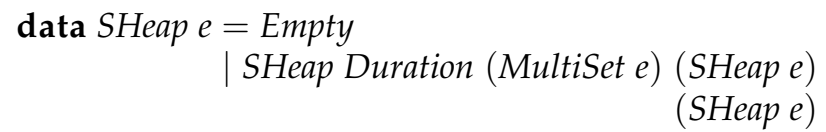


The events are stored in a multiset MultiSet, the Duration member is the time distance that separates the events in the node from his father, or the implicit pre marker of the tile for the root. In Figure 13 are depicted the same tiles as in Figure 9. The duration is represented by a dotted line between the two synchronization points, and the event is attached to the implicit pre marker of the tile (at time o).

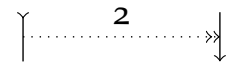

(a) The tile delay 2

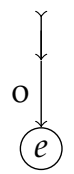

(b) The tile event $e$
Figure 13: Two simple heaped tiles

In Figure 14, we can see the correspondence between zigzag and heaped representations (same position on the horizontal axis means same time). In the later case, the syntactic composition of the tile is lost but the events are partially ordered.

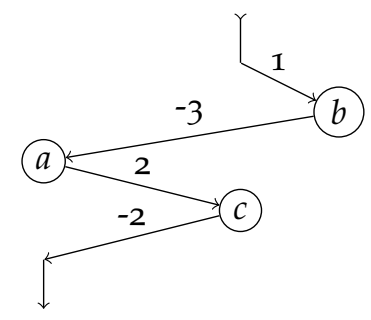

(a) Zigzag

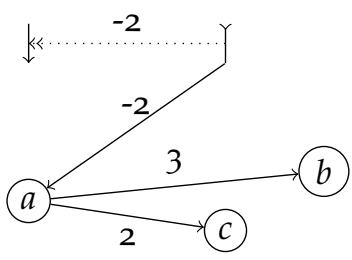

(b) Heaped

Figure 14: Two representations of the tile delay $1 \%$ event $b \%$ delay $(-3) \%$ event a $\%$ delay $2 \%$ event c $\%$ delay $(-2)$

\subsection{High Level Implementation of the Primitives}

The primitives introduced in 3.3 are implemented as follows:

$$
\begin{aligned}
& \text { delay } d=\text { Tile } d \text { Empty } \\
& \text { event } e=\text { Tile } 0 \text { (SHeap } 0 \text { (singleton e) Empty Empty) } \\
& \quad-- \text { singleton e is a multiset containing only e } \\
& (\%)\left(\text { Tile } d_{1} s_{1}\right)\left(\text { Tile } d_{2} s_{2}\right)= \\
& \text { Tile }\left(d_{1}+d_{2}\right)\left(\text { mergeSH } s_{1}\left(\text { shiftSH } d_{1} s_{2}\right)\right) \\
& \text { dur }\left(\text { Tile } d_{-}\right)=d
\end{aligned}
$$

The delay $d$ function returns a tile of duration $\mathrm{d}$ with no events, the event $e$ function returns a tile of $\mathrm{du}-$ ration $o$ and a single event at time distance o of the pre marker of the tile.

The mergeSH function is a binary operator that merges two heaps by preserving time distances, every event of each operand will be at the same time distance in the resulting tree. And the shiftSH function takes as parameter a duration and a heap, and shift all the timed events of the heap by the duration.

The implementation of composition is based on the property:

$$
t_{1} \% t_{2} \equiv \text { re } t_{1} \% \text { delay }\left(\text { dur } t_{1}\right) \% t_{2}
$$

The sole SHeap of the tile delay (dur $\left.t_{1}\right) \% t_{2}$, is computed by shiftSH $d_{1} s_{2}$ (every events of $t_{2}$ are preceded by a delay of $\left.\left(d u r t_{1}\right)\right)$. Then, the events of $t_{2}$ shifted are merged by the ones of $t_{1}$, and the duration of the resulting tile is the sum of the durations of $t_{1}$ and $t_{2}$. The tiled product is depicted in Figure 15 .

\subsection{Additional Primitives}

The rendering primitive are simple as well, all of the relative complexity of the implementation is hidden in the heap's primitives. Here the implementation of a few more primitives:

$$
\begin{aligned}
& \text { firstD } \left.\left(\text { Tile } \text { S SHeap }_{-}{ }_{--}\right)\right)=\text {Just } d \\
& \text { firstD }{ }_{-}=\text {Nothing }
\end{aligned}
$$




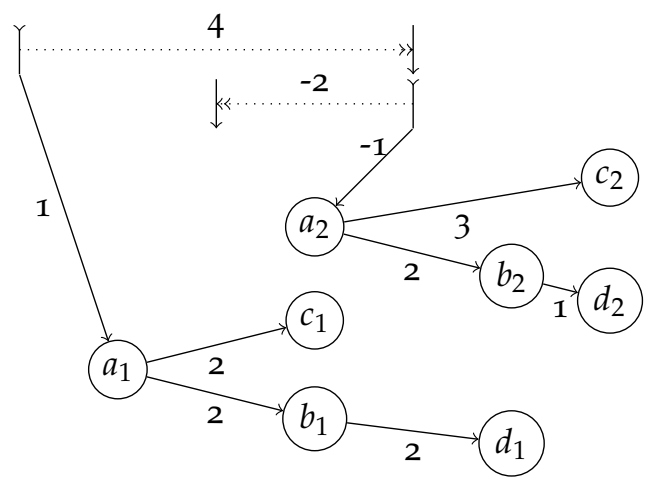

(a) The synchronization points are aligned (the heap of the second operand is shifted)

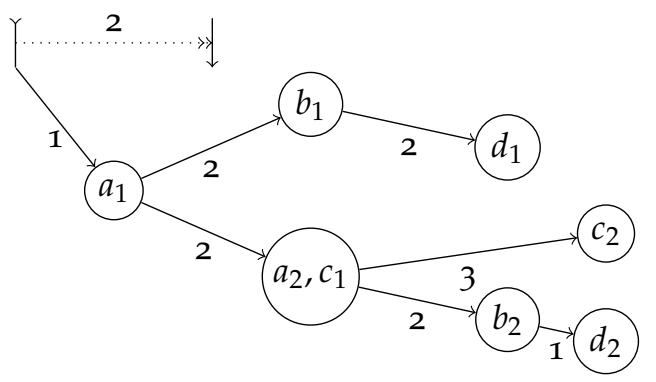

(b) The heaps and the synchronization data are merged

Figure 15: Tile composition

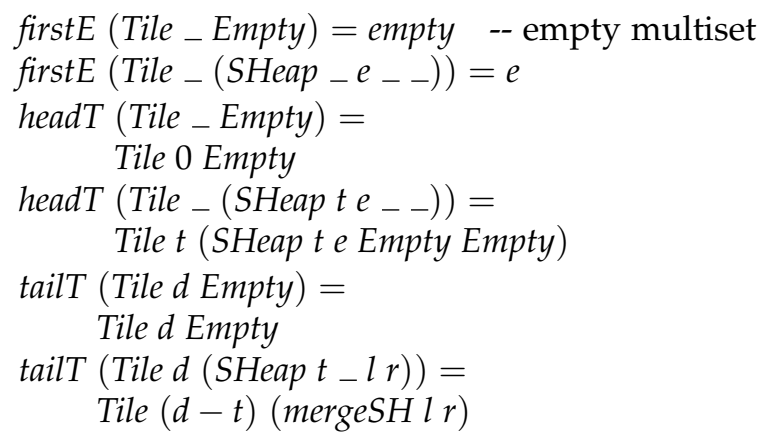

The tailT function removes the first events, and then computes a new heap containing all the remaining events.

A very important improvement with this tile representation is that the reset of a tile is defined even when the duration of this tile is undefined, as we can implement it by:

$$
\text { re }\left(\text { Tile } \_h\right)=\text { Tile } 0 h
$$

\subsection{Low Level Implementation}

A skew heap [7] is a very simple data structure that can be implemented in Haskell with less than 10 lines of code, it have nice algorithmic properties, and as it is a good base for an implementation of a priority queue, we can use it to implement TPTM as well. It is a binary tree where the nodes are labeled by elements of an ordered set (times for instance). It allows a quick access to the smallest element of the heap, and a quick merge of two heaps. Here, to store the events we uses a slightly modified skew heaps, the difference with the usual ones is that the nodes carry delays and not absolutes times.

The set of timed event is coded by a binary tree. In an expression of the form

$$
n=\text { SHeap d mse lchild rchild }
$$

where $d$ is the time distance between the events in the multiset mse and the event embedded by the father of $n$, or the implicit pre synchronization of the tile when $n$ is the root.

The invariant of the heap is:

- the duration carried by every node except the root is strictly positive,

- a node carry at least one event,

- the total delay to an event is the sum of all delays from the root to the node carrying this event.

The implementation of shiftSH is pretty straightforward. It is an $\mathcal{O}(1)$ operation: as the labels on the nodes are relatives, shifting a node shifts all its subtree. With absolutes labels we would have an $\mathcal{O}(n)$ operation. 
shiftSH :: Duration $\rightarrow$ Sheap $e \rightarrow$ SHeap e

shiftSH d Empty = Empty

shiftSH $d$ (SHeap $d^{\prime}$ e $\left.l r\right)=$ SHeap $\left(d+d^{\prime}\right)$ e $l r$

Finally, the implementation of mergeSH is given by:

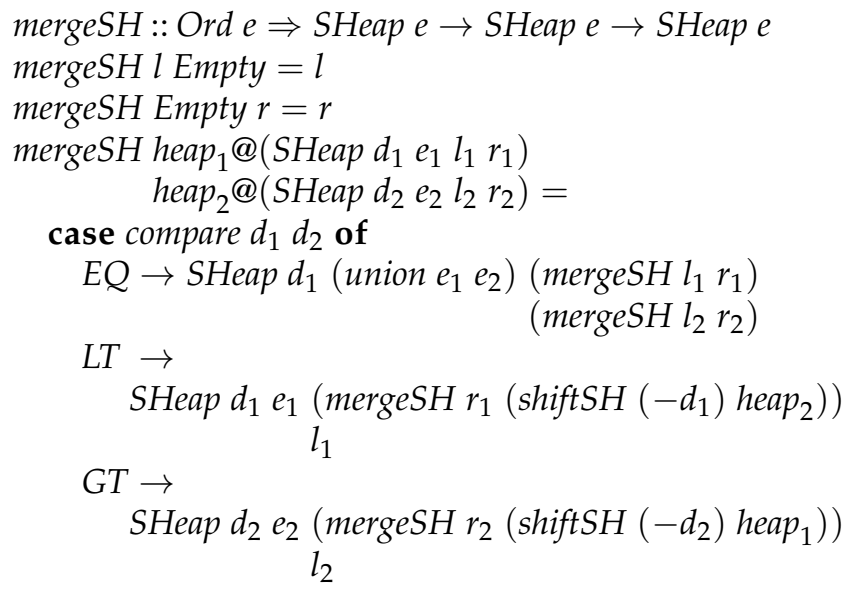

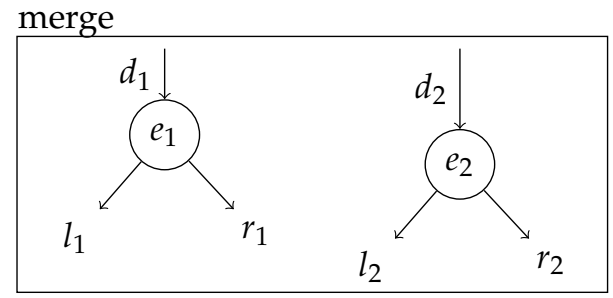

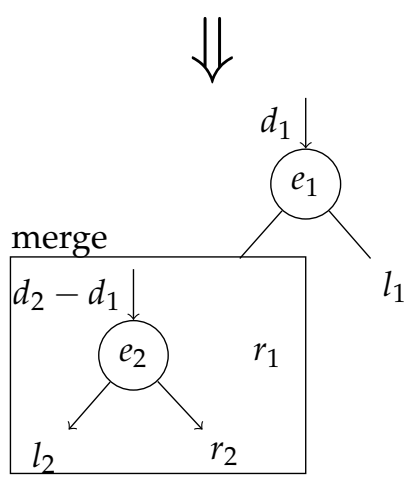

Figure 16: Heaps merge: case $d_{1}<d_{2}$
We merge the trees following their rightmost paths, and swap the right and the left child in the resulting tree. This case is depicted in Figure 16. By this mean, the tree tends to grow "from the inside" rather than the rightmost path.

If the roots of the two tree we want to merge have the same delay, we do not increase the resulting tree size and we merges their events instead, otherwise we would have a o delay and violate our invariant. This case is depicted in Figure 17. The fact that we forbid null delay is reducing the tree size, but we could as well allow it and then treating this special case in the headT function rather than in the merge function. We can use mergeSH over infinite heaps because it is lazy on the childs of its parameters.

\section{Complexity Analysis}

In this section we will study space and time complexity of a syntactic implementation and the heaped implementation. We define the size of a tile by $n=n_{e}+n_{d}$ where $n_{e}$ is the number of events and $n_{d}$ is the number of delays in it.

\subsection{Syntactic Complexity}

A syntactic implementation suffer from two algorithmic problems, about time and space complexity.

Firstly, there is no mechanism to balance the structure built. The complexity of the in-time rendering depends on the way the tile has been constructed. For instance, a call to first $E$ is linear on a tile that has been constructed starting "from the end", because the first events are in the bottom of the syntactic structure. In general, all operation are in $\mathcal{O}(n)$, where $n$ is the size of the tile.

Secondly, given a tile $t$ of any event type, there is no bound to the size of a tile $t^{\prime}$ such that $t \equiv t^{\prime}$. We can have an arbitrary number of delays because of the property delay $(a+b) \equiv$ delay $a \%$ delay $b$. With the implementation described in 3.1, the space complexity is $\mathcal{O}\left(n_{e}+n_{d}\right)$. 


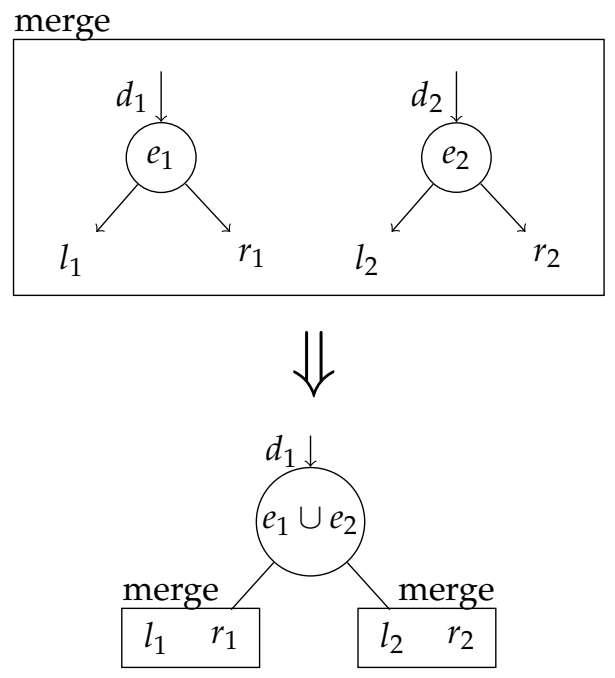

Figure 17: Heaps merge: case $d_{1}=d_{2}$

\subsection{Heaped Complexity}

With the heaped implementation and assuming that Duration have $\mathcal{O}(1)$ space complexity (for instance when a bounded number of delay values are used), the space complexity of a tile with $n_{e}$ events and $n_{d}$ delays is $\mathcal{O}\left(n_{e}\right)$ since the invariant of the heap imply $n_{d}=\mathcal{O}\left(n_{e}\right)$.

It can be proven [7] that mergeSH have an amortized complexity of $\mathcal{O}(\log n)$. Hence, both $\%$ and tailT have a logarithmic time complexity and headT have a constant time complexity.

\section{Performances}

In this section, we will compare the Twisted implementation [5], and the one introduced in this paper. In all the following measures, we simulate the rendering of a tile by recursively extracting all the first events of the tile. We use GHC 7.8.4 with no runtime tuning on an AMD Opteron 6174 (2.2 GHz). More specifically, we will benchmark the normalization of tiles defined as follow:
tileL $0=$ delay 0

tileL $n=($ delay $1 \%$ event ()$) \%$ tileL $($ pred $n)$

tileR $0=$ delay 0

tileR $n=$ tileR $($ pred $n) \%($ delay $1 \%$ event ()$)$

parallelTile t $n=$ foldl $(\%)$ (delay 0) (replicate $n($ re $t)$ )

tileLeft $=$ parallelTile (tileL 2000) 16

tileRight $=$ parallelTile $($ tileR 2000) 16

The performance of the normalization of a tile is very sensitive to its expression with the syntactic representation. The tile $R$ function generate a worst case for syntactic representation (parallelTile do not).

Due to the space complexity described in section 5.1, the extraction of the first events of a tile does not guaranty that the remainder is smaller than the original tile. The implementation described in [5] suffer from this, the number of delays in tailT $t$ increases. This is the reason why in Figure 18 the reduction time of firstE (tailT $\left.{ }^{n} t\right)$ is proportional to $n$.

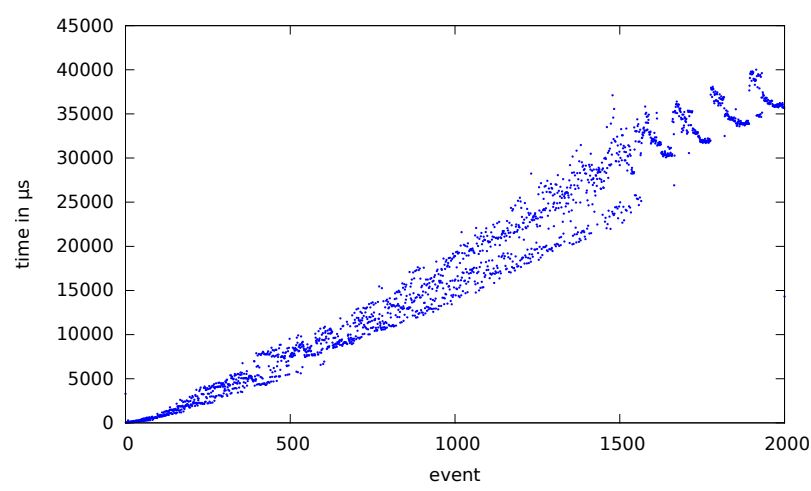

Figure 18: Normalization of tileLeft with the Twisted implementation

Thanks to the property delay $a \%$ delay $b \equiv$ delay $(a+b)$, we can always merge two consecutive delays and then reduce the tile size. By modifying the implementation of the \% operator in Twisted to guaranty that we never have two consecutive delays, we have a constant time call to firstE. In Figure 19 we see that the patched Twisted implementation can perform an in-time on-the-fly rendering on 
a left parenthesized tile. The very first call to firstE is longer because it trigger the evaluation of the tile.

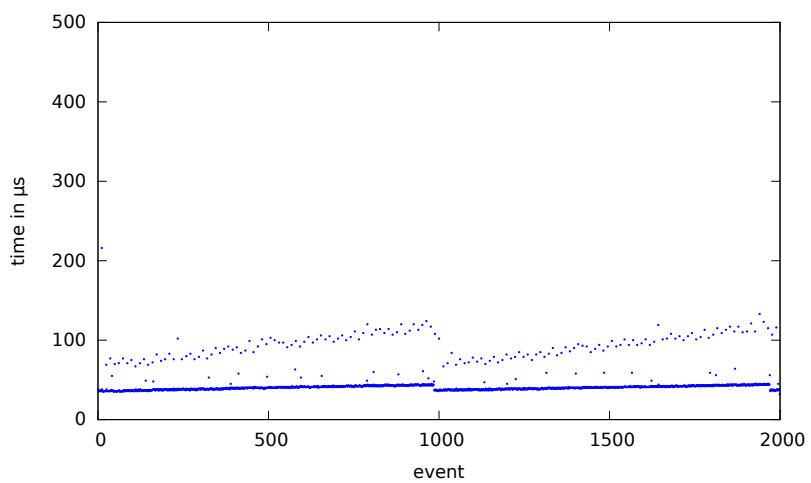

Figure 19: Normalization of tileLeft with the patched Twisted implementation

But the Twisted implementation stay very sensitive to the expression of the tile. In Figure 20 we see the normalization of the same tile but right parenthesized. The first events are the deepest leafs of an imbalanced syntactic tree, hence the reduction time of first $E$ is proportional to the size of the tile.

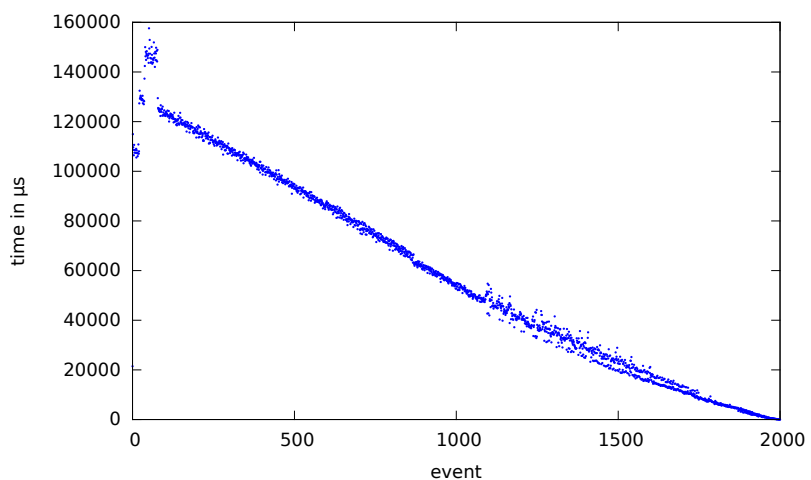

Figure 20: Normalization of tileRight with the patched Twisted implementation

The performance of the Heaped implementation performing the normalization of the same tile as in Figure 20 (a tile constructed "backward") is shown in Figure 21. The average call to first $E$ is less than $50 \mu \mathrm{s}$. The garbage collector periodically cause longer execution time. Because of the complicated complexity analysis of the skew heaps and the impact of the lazy semantics, a worst case for the Heaped implementation is yet to find.

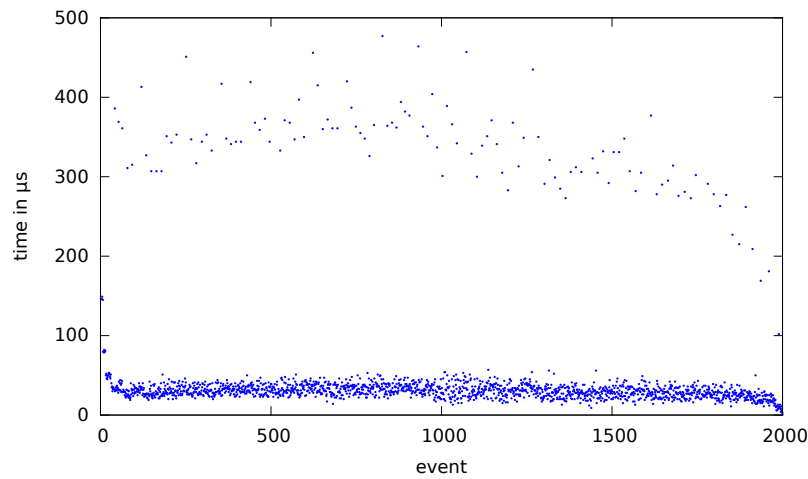

Figure 21: Normalization of tileRight with the new Heaped implementation (some few points are between 500 and $5000 \mu \mathrm{s})$

The normalization of a left parenthesized tile with the Heaped implementation is depicted in Figure 22. The first call to firtsE last $1400 \mu \mathrm{s}$, the average is $6 \mu \mathrm{s}$.

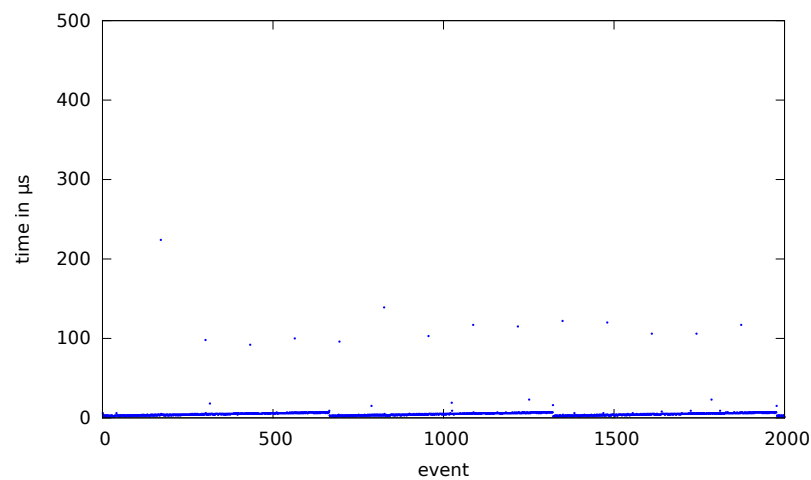

Figure 22: Normalization of tileLeft with the new Heaped implementation (some few points are between 500 and $1500 \mu \mathrm{s})$ 


\section{$7 \quad$ Infinite Tiles}

As the lazy semantics of Haskell allows the programmer to write infinite data structures such as lists, we would like to be able to write infinite tiles. In this section we study the possibility of representing and playing an infinite tile. Such a tile that may results from an equation of the form $x=m \%$ re $x$ [4]. Because we are interested in relevant tile from a musical point on view, we will consider only the tiles with a finite number of events at each time, and where all delay $d_{i}$ between consecutive set of events verify for all $i$ : $0<m \leqslant d_{i} \leqslant M$ for some $m$ and $M$. This mean in particular that we can not have an infinite number of events in a finite time interval.

\subsection{Syntactic Implementation}

With the syntactic representation, we observe that it is impossible to build such an infinite tile with a finite duration. Because the duration of the tile is the sum all the delays that composes it, and a infinite tile have an infinite number of positive delays, we would need to add an infinite number of negative delays to make the sum finite. We can write a function that compute an potentially infinite tile from a potentially infinite list for instance, but it will suffer from a memory leak because of the infinite number of negative delays accumulated needed to have a finite duration. The Figure 23 depict such a tile.

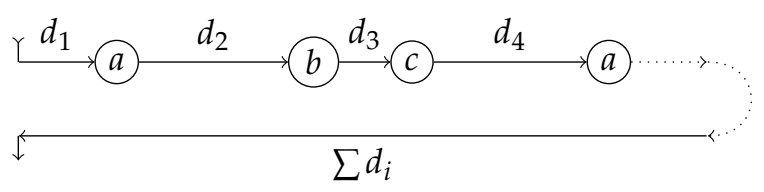

Figure 23: A potentially infinite zigzag tile

\subsection{Heaped Implementation}

As in tiled Euterpea, it is possible to represent an infinite tile with the Heaped implementation. The reason is that the set of SHeap e with empty right childs is isomorphic to the list type [(Duration, MultiSet e $)]$ where all the durations in it are positives except maybe the first one and all multisets are non empty. Hence, we can build infinite tiles given an infinite list.

We define a function sToT as Stream To Tile, that takes a potentially infinite list and returns an infinite tile of finite duration that encode this list. Such a tile is depicted in Figure 24 .

$$
\begin{aligned}
& \text { sToT } l=\text { Tile } 0(\text { lToSH } l) \\
& l \text { ToSH }::[(\text { Duration, MultiSet } e)] \rightarrow \text { SHeap e } \\
& \text { lToSH }[]=\text { Empty } \\
& \text { lToSH }\left((d, e):\left(0, e^{\prime}\right): l\right)=l \text { ToSH }\left(\left(d, \text { union e } e^{\prime}\right): l\right) \\
& \quad-- \text { we don't want o's } \\
& l \text { ToSH }((d, e): l)=\text { SHeap de }(\text { lToSH l) Empty }
\end{aligned}
$$

The $I T o S H$ function computes a SHeap from the list while reducing o delays, and the $s T o T$ function returns a null duration Tile with this heap.

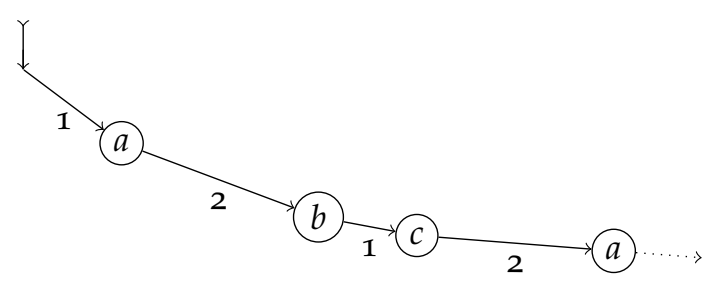

Figure 24: An infinite heaped tile

Unlike the implementation in 7.2 , here we can build and render an infinite tile with no memory leaks.

\section{Encoding PTM into TPTM}

As a consequence of the possibility to compute infinite Heaped tile, we can write conversion function from infinite PTM to TPTM. This implementation suffers from a memory leak when the PTM converted is have an infinite duration.

We use this simple definition for PTM, and a Event to encode temporal media in term of events: 


$\begin{aligned} & \text { data Media } a=\text { Prim Duration a } \\ & \mid \text { Wait Duration } \\ & \mid \text { Media } a:+ \text { Media } a \\ & \text { Media } a:=: \text { Media } a \\ & \text { data Event } a=\text { On } a \mid \text { Off } a \text { deriving }(E q, \text { Ord })\end{aligned}$

The constructors Prim $d a$ represents a media $a$ during a time $d$, Wait $d$ is a delay, and the remaining constructors are pretty straightforward. In TPTM, we encode a media $a$ for a duration $d$ by two event separated by a time $d$ marking the beginning and the end of $a$.

The tiles involved here have the property:

$(P)$ All the events embedded are between the two synchronization marks: There is no event before the pre marker, and no event after the post marker.

A TPTM encoding a PTM is a well parenthesized sequence of event On $a$ and Off $a$. We define a function $m T o T$ as Media To Tile that computes such a tile:

$$
\begin{aligned}
& m T o T:: \text { Ord } a \Rightarrow \text { Media } a \rightarrow \text { Tile (Event } a \text { ) } \\
& m \text { ToT }(\text { Prim } d a)= \\
& \text { event (On a) \% delay d \% event (Off a) } \\
& \text { mToT }(\text { Wait } d)=\text { delay } d \\
& \operatorname{mToT}\left(m_{1}:=: m_{2}\right)=\operatorname{parM}\left(\text { mToT } m_{1}\right)\left(\text { mToT } m_{2}\right) \\
& \operatorname{mToT}\left(m_{1}:+: m_{2}\right)=\operatorname{seqM}\left(\text { mToT } m_{1}\right)\left(\text { mToT } m_{2}\right)
\end{aligned}
$$

The seqM and par $M$ functions are the tiled implementation of $:+:$ and $:=$ : respectively, in order to be able do deal with infinite tiles, these functions can not be implemented naively. The seq $M$ function works under the hypothesis that both operands verify $(P)$.

$$
\begin{aligned}
& \text { parM }\left(\text { Tile } d_{1} h 1\right)\left(\text { Tile } d_{2} h 2\right)= \\
& \text { Tile }\left(\text { max } d_{1} d_{2}\right)(\text { mergeSH } h 1 \text { h2 }) \\
& \text { seqM }\left(\text { Tile } d_{1} h 1\right) \sim\left(\text { Tile } d_{2} h 2\right)= \\
& \text { Tile } \left.\left(d_{1}+d_{2}\right)\left(\text { lazyMergeSH h1 (shiftSH } d_{1} h 2\right)\right)
\end{aligned}
$$

The implementation of parM is pretty straightforward, we notice that parM is lazy on durations, and
mergeSH can handle infinite heaps, so such a function suit its purpose. However, the implementation of seqM is less obvious because it have to be lazy on its second parameter in order to be able to handle an infinite PTM such as $m_{1}:+:\left(m_{2}:+:\left(m_{3}:+:\left(m_{4} \cdots\right.\right.\right.$

The first unwanted strictness come from the fact that in Haskell, pattern matching in case expression are strict by default. Without the "lazy-pattern" tilde, when seqM $a b$ is evaluated, it will trigger the evaluation of $a$ and $b, b$ can also be of the form seqM $c d$, hence, it will trigger an infinite computation. Such a pattern matching cannot fail because Tile $e$ have a single constructor. We could as well made the program lazy on the second operand by other mean, but for the sake of simplicity, we use the $\sim($ pattern $)$ syntax.

The second unwanted strictness comes from mergesH because it needs to evaluate both its operand in order to see from which one the first event of the resulting tree comes from. We use the hypothesis $(P)$ to implement a lazier merge.

$$
\begin{aligned}
& \text { lazyMergeSH }:: \text { SHeap e } \rightarrow \text { SHeap e } \rightarrow \text { SHeap e } \\
& \text { lazyMergeSH Empty } h=h \\
& \text { lazyMergeSH }(\text { SHeap d e l } r) h= \\
& \text { SHeapd e (lazyMergeSH } r(\text { shiftSH }(-d) h)) l
\end{aligned}
$$

The lazyMergeSH function is lazy on the second parameter and works only under the hypothesis that the first event of the second heap is after the last event of the first heap. The algorithm is exactly the same as mergeSH, but by hypothesis we are always in the $L T$ case.

The $m T o T$ function build a tile of the duration of the media, such a duration can be undefined in case of infinite PTM such as $m$ in $m=m_{1}:+: m$. It follows that one will prefer the use of the function $m T o R e T$ which returns the reset of that tile, of duration 0.

$$
\begin{aligned}
& m \text { ToReT }:: \text { Ord } a \Rightarrow \text { Media } a \rightarrow \text { Tile (Event } a) \\
& m \text { ToReT }=\text { re } \circ \text { mToT }
\end{aligned}
$$

Such an implementation suffers from a memory leak if the PTM converted is infinite. Because the 
function $m T o T$ will compute without evaluating the duration of the resulting tile, which can be infinite.

\section{Rendering}

The first $E$, first $D$ and tailT performs an abstract rendering. In order to make more concretes experiments around TPTM, two players have been written. The first one is a MIDI player, and the second one an audio player.

These two players relies on the sound server JACK that provides a real-time engine and primitive to send both MIDI and audio. The input of JACK is bufferized, this conveniently loosen the real-time constrains imposed to the Haskell runtime.

\subsection{MIDI Player}

The MIDI player takes as input a tile of MIDI messages and feed the JACK server by performing an on-the-fly normalization of the tile. The firstE function performs an agnostic accumulation of the MIDI events, the player relies on downstream components to compute the semantic of simultaneous events.

This player have been used to play standard MIDI files. Such a file can be seen as a list of parallel tracks (usually one per instrument), where a track is a sequence of delays and MIDI events. Hence, we encode a MIDI file by a tile of the form re $t_{1} \%$ re $t_{2} \%$ re $t_{3} \%$... where each $t_{n}$ is a positive tile encoding of the $n$th MIDI track.

\subsection{Audio Player}

We implemented a player that is capable of reading and playing audio tile as well. A digital audio stream is a continuous signal discretized at a constant framerate. For performance reasons, rendering a tile with one event per audio frame was not feasible. Thus, an audio tile is based on the granular synthesis technique. The audio events are "grain", some few milliseconds of audio stream. These grains overlap and are placed in an envelope in order to avoid artifacts.
Thanks to this technique, we can easily change the tempo without modifying the pitch, as it suffice to modify the delays in tile separating the grains. However, significant speed change generate a lot of artifacts.

A reduction have to be performed on the events returned by first $E$, from a multiset of grains we compute a vector of audio frames.

These experiments open the way to musical experimentation. We are currently working on a player that can simultaneously read both MIDI and audio.

\section{Conclusion}

The implementation proposed in this paper have good algorithmic properties and can handle infinite temporal media, just as Tiled Euterpea could [4].

In further research we would like to extend TPTM model in order to handle interactive temporal media. In particular, we are interested in being able to describe some synchronizations between the rendering of a tile and an external stream of events (the music played by a musician for instance). We are also interested in expressing complex interactive programs with conditional renderings.

The tree structure of our implementation seems to be adapted to this extension as it induces a partial order between the events that is compatible with the temporality of those events. This tree describes a non-determinism between the order of the events to be played that is resolved by the normalization. An arbitrary arity tree could express a nondeterminism between the order of external events that would be resolved by the monitoring of external events. Of course, this would imply exploring the possible links with other known approaches such as, in particular, Functional Reactive Programming approach.

\section{References}

[1] T. Bazin and D. Janin. Flux média tuilés polymorphes: une sémantique opérationnelle en 
Haskell. 2015.

[2] P. Hudak. An algebraic theory of polymorphic temporal media. In Proceedings of PADL'04: 6th International Workshop on Practical Aspects of Declarative Languages, pages 1-15, 2004.

[3] P. Hudak. The Haskell School of Music : From signals to Symphonies. Yale University, Department of Computer Science, 2013.

[4] P. Hudak and D. Janin. Tiled polymorphic temporal media. In ACM Workshop on Functional Art, Music, Modeling and Design (FARM), pages 49-6o. ACM Press, 2014.
[5] P. Hudak and D. Janin. From out-of-time design to in-time production of temporal media. Technical report, LaBRI, Université de Bordeaux, 2015.

[6] P. Hudak, T. Makucevich, S. Gadde, and B. Whong. Haskore music notation - an algebra of music. Journal of Functional Programming, 6(3):465-483, May 1996.

[7] D. D. Sleator and R. E. Tarjan. Self adjusting heaps. SIAM J. Comput., 15(1). 